\title{
Percepção de justiça e COMPORTAMENTO DE CIDADANIA ORGANIZACIONAL: UM ESTUDO NA PERSPECTIVA DO SISTEMA DE MENSURAÇÃO DE DESEMPENHO ESTRATÉGICO*
}

\author{
Ilse Maria Beuren ${ }^{* * *}$ \\ Stella Maris Lima Altoé \\ Delci Grapégia Dal Vesco
}

* Doi: 10.11144/Javeriana.cao28-51.pjcc. Este artigo é resultado da pesquisa "Percepção de justiça organizacional dos controllers de empresas com sistemas de remuneração por recompensa", financiado pelo Conselho Nacional de Desenvolvimento Científico e Tecnológico (CNPq) desde 2011 até 2013. Este artigo foi recebido em 09/05/2015 e aprovado em 11/11/2015. Sugestão de citação: Beuren, I. M.; Altoé, S. M. L., e Dal Vesco, D. G. (2015). Percepção de justiça e comportamento de cidadania organizacional: um estudo na perspectiva do Sistema de Mensuração de Desempenho Estratégico. Cuadernos de Administración, 28 (51), 133-157. http://dx.doi.org/10.11144/Javeriana.cao28-51.pjcc

* * Doutorado em Controladoria e Contabilidade pela Universidade de São Paulo (USP), Brasil, 1995. Professora do Programa de Pós-graduação em Ciências Contábeis da Universidade Federal de Santa Catarina (UFSC), Florianópolis, Santa Catarina, Brasil.

E-mail: ilse.beuren@gmail.com

*** Doutoranda no Programa de Pós-graduação em Contabilidade da Universidade Federal do Paraná (UFPR), Curitiba, Paraná, Brasil.

E-mail: stella.altoe@gmail.com

**** Doutora em Contabilidade e Administração pela Universidade Regional de Blumenau (FURB), Blumenau, Santa Catarina, Brasil, 2013. Professora do Programa de Pós-Graduação em Ciências Contábeis da Universidade Estadual do Oeste do Paraná (UNIOESTE), Cascavel, Paraná, Brasil.

E-mail: delcigrape@gmail.com 


\section{Percepção de justiça \\ e comportamento de cidadania organizacional: um estudo na perspectiva do sistema de mensuração de desempenho estratégico}

Percepción de justicia y comportamiento de ciudadanía organizacional: un estudio en perspectiva del sistema de medición de desempeño estratégico

\section{RESUMO}

O estudo objetiva verificar a relação entre justiça organizacional e comportamento de cidadania organizacional de empregados na perspectiva do Sistema de Mensuração de Desempenho Estratégico. Uma survey online foi realizada tendo como amostra 105 empregados de uma cooperativa de crédito. Para testar as hipóteses replicadas de Burney, Henle e Widener (2009), utilizou-se modelagem de equações estruturais. Os resultados mostram que somente a relação entre o grau de validade técnica do sistema e a justiça distributiva não foi significante. Assim, conclui-se que os resultados assemelham-se aos de Burney, Henle e Widener (2009), mas instigam pesquisas para explicar a hipótese não confirmada.

Palavras-chave: desempenho estratégico, justiça organizacional, comportamento de cidadania organizacional.

Classificação JEL: M14, M41

\section{RESUMEN}

El propósito de este estudio es investigar la relación entre la justicia organizacional y el comportamiento de la ciudadanía organizacional de los empleados desde la perspectiva del Sistema de Medición del Desempeño Estratégico. Se llevó a cabo una encuesta online teniendo como muestra 105 empleados de una cooperativa de crédito. Para probar las hipótesis replicadas de Burney, Henle e Widener (2009), se utilizó el modelo de ecuaciones estructurales. Los resultados muestran que sólo la relación entre el grado de validez técnica del sistema y la justicia distributiva no fue significativa. Así, se concluye que los resultados son similares a los de Burney, Henle y Widener (2009), y para futuras investigaciones se propone explicar la hipótesis sin confirmar.

Palabras clave: desempeño estratégico, justicia organizacional, comportamiento de ciudadanía organizacional.

Clasificación JEL: M14, M41

Perception of organizational justice and citizenship behavior: A study in the perspective of the strategic performance measurement system

\section{ABSTRACT}

The goal of this study is to investigate the relationship between organizational justice and organizational citizenship behavior of employees from the perspective of the Strategic Performance Measurement System. An online survey was conducted using 105 employees of a credit cooperative as its sample. To test the hypotheses replicated from Burney, Henle e Widener (2009), structural equation modeling was used. The results show that only the relationship between the degree of system technical validity and distributive justice was not significant. Thus, it is concluded that the results are similar to Burney, Henle and Widener (2009), and it is proposed that, for future studies, the unconfirmed hypothesis should be explained.

Keywords: Strategic performance, organizational justice, organizational citizenship behavior.

JEL Classification: $\mathrm{M}_{14}, \mathrm{M}_{41}$ 


\section{Introdução}

A mensuração do desempenho é uma das funções fundamentais da gestão organizacional. Nessa perspectiva, o Sistema de Mensuração de Desempenho Estratégico (Strategic Performance Measurement System: SPMS) se propõe a avaliar o desempenho e as contingências ambientais, realizando os ajustes necessários à estratégia empresarial (Chaneta, 2007). A concepção de um SPMS tem como foco principal contribuir para a comunicação da estratégia empresarial aos seus empregados. A configuração do SPMS contempla os aspectos financeiro, operacional e estratégico (Ittner, Larcker e Randall, 2003).

Depreende-se, assim, a relevância de as organizações identificarem a percepção dos empregados sobre os atributos que regem o SPMS (Burney, Henle e Widener, 2009), uma vez que o comportamento deles está vinculado à percepção que possuem do SPMS (Robbins, 2003). A percepção dos empregados sobre o SPMS está relacionada com a percepção de justiça organizacional, cujo propósito é embasar o julgamento deles quanto aos fatores que interferem no desempenho organizacional (Johnson, Selenta e Lord, 2006). Para esses autores, a percepção de justiça organizacional contempla aspectos relativos a satisfação dos empregados, comprometimento organizacional, comportamento de cidadania organizacional e desempenho no trabalho.

Diferentes vertentes de justiça organizacional são abordadas na literatura, destacando-se as justiças processual, distributiva e interacional (Colquitt, 2001). A justiça processual orienta-se pelas discussões sobre os procedimentos, como os utilizados na avaliação de desempenho organizacional (Leventhal, 1980; Leventhal, Karuza e Fry, 1980; Folger e Cropanzano, 1998; Rego, 2002; Sotomayor, 2007). A justiça distributiva analisa a percepção dos empregados sobre salário, promoç̃̃es, distribuição de resultados (Rego, 2002). Já a justiça interacional refere-se à abordagem interpessoal, ressaltando os aspectos de tratamento de gestores, antes e/ou após a implantação de uma avaliação estratégica (Colquitt, Conlon, Wesson, Porter e Ng, 2001).

Neste estudo, a justiça organizacional é analisada nas vertentes processual e distributiva. A justiça processual é analisada à luz dos procedimentos relativos ao SPMS (Burney, Henle e Widener, 2009). E a justiça distributiva focaliza a percepção dos empregados nos aspectos vinculados à remuneração. A justiça interacional não faz parte do escopo deste estudo, pois o seu alvo é a percepção que o empregado tem do gestor, a partir da implementação do SPMS, diante dos parâmetros do sistema e sua relação com a remuneração, aspectos direcionados ao desempenho organizacional. 
As justiças processual e distributiva encontram-se imbricadas no escopo da empresa (Cropanzano e Ambrose, 2001). Por sua vez, a percepção dos empregados, frente a esses aspectos de justiça organizacional, afeta o comportamento de cidadania organizacional, que diz respeito às ações espontâneas realizadas pelos empregados, com o propósito de contribuir aos resultados organizacionais (Burney, Henle e Widener, 2009).

Na perspectiva do comportamento de cidadania organizacional, orientado para as ações humanas voluntárias, os indivíduos agem em função de sua vontade própria; portanto, sem vinculação com recompensas (Organ, 1998; Çinar, Karcioglu e Aliogullari, 2013). Tais atitudes contribuem ao desempenho da função para além dos papéis estabelecidos, denominados de comportamento "extrapapel" (Williams e Anderson, 1991; Van Dyne, Graham e Dienesch, 1994). 0 comportamento de cidadania organizacional favorece 0 alcance da eficácia (Podsakoff e Mackenzie, 1997) à medida que contribui para o desempenho organizacional (Çinar, Karcioglu e Aliogullari, 2013).

Diante do exposto, a problemática refere-se à concepção equivocada do projeto ideal de incentivos (Locke e Latham, 1990; Beer e Cannon, 2004). Presume-se que, quando o sistema de avaliação de desempenho não apresenta parâmetros claros e conscisos, a percepção de justiça organizacional e o comportamento de cidadania organizacional dos empregados tendem a ser prejudicados e avaliados como negativos.

Assim, a questão norteadora da presente investigação é: qual a relação entre a percepção da justiça organizacional e o comportamento de cidadania organizacional de empregados na perspectiva do Sistema de Mensuração de Desempenho Estratégico? Para isso, o objetivo do estudo é verificar a relação entre a percepção de justiça organizacional e o comportamento de cidadania organizacional na perspectiva do Sistema de Mensuração de Desempenho Estratégico dos empregados de uma cooperativa de crédito.

0 estudo justifica-se pela relevância de as organizações considerarem as ações individuais dos empregados para o alcance dos objetivos globais estabelecidos (Schiemann e Lingle, 1997; Otley, 1999). A percepção dos empregados sobre o SPMS, sob a ótica da justiça organizacional e do comportamento de cidadania organizacional, reflete-se nos resultados organizacionais (Burney, Henle e Widener, 2009).

Embora este estudo seja uma réplica da pesquisa de Burney, Henle e Widener (2009), diferencia-se do original em função de este ter sido aplicado em bancos, enquanto esta pesquisa é realizada em uma cooperativa de crédito. As cooperativas se diferenciam das 
demais formas empresariais; primeiro, porque o lucro não é a intenção inicial; segundo, ao final do exercício, os recursos gerados são tratados como sobras operacionais, que serão destinadas aos cooperados (Bialoskorski Neto e Marques, 1998). Presume-se que essas particularidades resultem em diferenças no campo de estudo e nos resultados da pesquisa.

A seleção de uma organização desse setor também decorre da importância do cooperativismo para a economia brasileira. Conforme estatísticas da Organização das Cooperativas Brasileiras (OCB), o cooperativismo atua em expansão no Brasil, sobretudo no quesito geração de empregos. As perspectivas indicam que cerca de 353 mil empregos sejam oferecidos até o final de 2016. Além disso, o setor, em 2011, registrou USD\$ 6,1 bilhões em exportações, sendo o complexo sucroalcooleiro o que apresentou maior destaque, exportando cerca de USD\$2,2 bilhões, seguido pelo complexo de soja, com USD\$1,3 bilhão.

Busca-se ainda contribuir para a escassez de pesquisas que abordam sobre os efeitos do SPMS no desempenho organizacional. Estudos anteriores investigaram aspectos de subjetividade no SPMS (Ittner, Larcker e Meyer, 2003; Gibbs, Merchant, Van Der Stede e Vargus, 2004), mas são poucas as pesquisas direcionadas às características do SPMS. Além disso, a literatura sugere que não há uniformidade quanto às características do SPMS quando a finalidade é maximizar o desempenho organizacional (Burney, Henle e Widener, 2009).

\section{Referencial teórico}

0 Sistema de Mensuração de Desempenho Estratégico (SPMS) é o conjunto de métricas, financeiras e não financeiras, de curto e longo prazo, internas e externas, ex post e ex ante, que fundamentam o processo de tomada de decisão e que, após o processamento dos dados, fornecem informações quantificadas sobre o desempenho na forma de relatórios (Gimbert, Bisbe e Mendoza, 2010; Bisbe e Malagueno, 2012). É um sistema que traduz as estratégias organizacionais em resultados distribuíveis, combinando medidas estratégicas, financeiras e operacionais, capazes de mensurar o cumprimento das metas (Hall, 2008). 0 SPMS fornece informações financeiras e não financeiras, que permitem identificar as estratégias que apresentam maior potencial para atingir os objetivos da empresa, a lém de alinhar os processos de gestão definidos (Ittner, Larcker e Randall, 2003).

Constata-se benefícios que são proporcionados pelo SPMS para a performance organizacional (Crabtree e Debusk, 2008; Geuser, Mooraj e Oyon, 2009) à medida que interage com a comunicação, a execução e as estratégias traçadas pela organização (Garengo, Biazzo e 
Bititci, 2005). 0 SPMS ajuda a traduzir a estratégia organizacional em objetivos e formas de mensuração, de modo que esse processo de comunicação possa contribuir para que o gap entre a visão estratégica da empresa e a gestão das atividades operacionais seja minimizado (Geuser, Mooraj e Oyon, 2009; Bisbe e Malagueno, 2012).

Esse sistema também favorece a manutenção da competitividade da organização, considerando que as estratégias serão implementadas e compatíveis com a cadeia de valor (Chenhall, 2005). Aracioglu, Zalluhoglu e Candemir (2013) destacam que a mensuração do desempenho estratégico é vital para todas as organizações, e sua finalidade consiste em auxiliar os gestores a definirem e atingirem os objetivos e planos estratégicos, além de assessorar nas decisões críticas inerentes a esse ambiente.

0 dinamismo ambiental é um fator crítico, capaz de influenciar de forma significativa o efeito do SPMS no desempenho organizacional (Bisbe e Malagueno, 2012). Os autores explicam que, em ambientes estáveis, o SPMS apresenta efeitos positivos, pois os gestores conseguem acompanhá-los por meio da estratégia de reformulação dos procedimentos. Porém, em cenários dinâmicos, tal possibilidade é reduzida, considerando que a interferência de diversas atividades dificulta a alteração das rotinas estabelecidas.

Conforme Bisbe e Malagueno (2012), a mensuração do desempenho estratégico possibilita: 1) a implementação e reformulação da estratégia; 2) a comunicação dos principais objetivos e prioridades corporativas; 3 ) o alinhamento estratégico; 4) a melhoria dos processos e 5) o incentivo à inovação. 0 foco do SPMS é a melhoria dos processos internos e externos da organização, o que o torna relevante para maximizar o desempenho organizacional (Marinho e Cagnin, 2014). Além disso, fornece aos gestores a possibilidade de melhorar o alinhamento estratégico, pois seu reflexo, por meio de medidas financeiras e não financeiras, pode ser observado em toda a organização (Kolehmainen, 2010).

Duas importantes características do SPMS são apontadas por Kaplan e Norton (1996): 0 modelo estratégico causal e a validade técnica. Para esses autores, o modelo estratégico causal refere-se ao alinhamento das estratégias organizacionais para o alcance das metas estabelecidas. Por sua vez, a validade técnica consiste em medidas precisas, acessíveis, compreensíveis e disponibilizadas em tempo hábil. A fusão dessas duas características promoverá a percepção de justiça organizacional pelos empregados.

De acordo com Franco-Santos, Lucianetti e Bourne (2012), sob a ótica dos indivíduos o SPMS pode facilitar o desenvolvimento, a implementação e a avaliação das estratégias 
organizacionais, desde que o alvo seja as decisões e as ações individuais. Pode ainda impactar no processo de comunicação e fornecer informações que influenciam o modo como as pessoas pensam, agem e interagem. Portanto, interfere na rotina organizacional e nas práticas gerenciais, que, por sua vez, alteram o comportamento dos gestores.

Franco-Santos, Lucianetti e Bourne (2012) destacam que o desempenho do SPMS é moderado por contingências internas (experiência dos funcionários, orientação estratégica da organização, estrutura, sistemas de informação, cultura e estilo de gestão) e externas (concorrência, grau de incerteza do ambiente em que a organização opera). Portanto, quando o SPMS não é implantado adequadamente, pode aumentar os custos e a carga de trabalho, resultando em tensões internas. Pode também trazer preconceitos e percepções de injustiça ou subjetividade, quando utilizado para fins de avaliação de desempenho.

\subsection{Justiça organizacional e o SPMS}

Estudos sobre justiça organizacional originaram-se da Teoria da Equidade, desenvolvida por Strayce Adams. A Teoria da Equidade estabelece que os indivíduos são motivados a firmarem relações equitativas, com o propósito de evitarem situações injustas ou desbalanceadas. Além disso, os indivíduos confrontam seus resultados com o de outras pessoas e emitem percepções sobre a justiça dessas relações (Adams, 1965).

A justiça organizacional contribui para entender e predizer o comportamento organizacional (Hartman, Yrle e Galle Jr, 1999). Assmar, Ferreira e Souto (2005) explicam que a justiça organizacional refere-se à percepção de justiça por parte dos empregados no que concerne às relações de trabalho. A percepção de justiça organizacional desdobra-se em justiça processual, justiça distributiva e justiça interacional (Colquitt, 2001; Colquitt et al., 2001). Neste estudo, a atenção recai sobre as duas categorias iniciais de justiça organizacional, a processual e a distributiva, em função de sua interação com o SPMS.

Ajustiça processual focaliza os processos e discute a concepção de justiça sobre os meios que são utilizados para alcançar os fins; sua percepção por parte dos empregados fundamenta-se nos procedimentos utilizados para processos disciplinares e sistemas de avaliação de desempenho (Leventhal, 1980; Leventhal, Karuza e Fry, 1980; Folger e Cropanzano 1998; Rego, 2002; Sotomayor, 2007). Os empregados avaliam a justiça processual ao longo das dimensões: 1) avaliação dos padrões de desempenho da organização (Gilliland e Langdon, 1998); 2) estabelecimento de um sistema orientado ao alcance dos objetivos, 
em que os empregados estão engajados (Gilliland e Langdon, 1998) e 3) características do processo (Folger, Konovsky e Cropanzano, 1992).

Ressalta-se que a percepção de justiça processual é positiva quando os empregados apresentam percepção favorável sobre o processo de avaliação de desempenho, cuja visão contribui para que os empregados atribuam maior valor à organização e, consequentemente, melhora o desempenho organizacional (Folger e Cropanzano, 1998). A justiça processual, na perspectiva do SPMS, fundamenta-se na Teoria de Controle de Processos, ou seja, que a percepção dos empregados seja de maior controle sobre os procedimentos utilizados para fundamentar a tomada de decisão (Erdogan, 2002).

A justiça distributiva tem origem no estudo de Homans (1961) e na Teoria de Troca no Comportamento Social, que considera duas abordagens de investigação. A primeira é que as recompensas devem ser proporcionais em relação aos custos, e a segunda prevê que os lucros devem ser proporcionais aos investimentos realizados (Homans, 1961). Portanto, o foco de análise da justiça distributiva é o resultado. Rego (2002) cita como exemplos de justiça distributiva o salário, a avaliação de desempenho, as promoções e a distribuição de lucros.

\subsection{Comportamento de cidadania organizacional}

0 conceito de cidadania organizacional foi introduzido na literatura por Organ (1998), que se refere ao comportamento individual discricionário dos membros da organização, o qual não é explicitamente reconhecido pelo sistema formal de recompensas, porém sua finalidade é promover o adequado funcionamento da organização. Nessa visão, há ligação direta entre comportamento de cidadania organizacional e desempenho organizacional.

0 ponto central de análise do comportamento de cidadania organizacional está nas relações entre os membros da organização, com vistas à otimização do desempenho organizacional (Popescu e Deaconu, 2013). Para Çinar, Karcioglu e Aliogullari (2013), ela configura-se como uma nova noção de análise de desempenho, representando uma conduta humana antiga de ação voluntária e ajuda mútua com nenhuma intenção de recompensa formal em troca. Para esses autores, as organizações precisam de empregados que realizam atividades além de suas funções habituais de trabalho e proporcionam desempenho que supere as expectativas. Portanto, comportamento de cidadania organizacional inclui ações de empregados que ultrapassem seus papéis definidos. 
0 comportamento de cidadania organizacional aumenta o desempenho em função de contribuir para: 1) a maximização da produtividade; 2) a redução da necessidade de destinar recursos específicos à manutenção de funções; 3 ) a melhoria da coordenação entre os membros que compõem o grupo de trabalho; 4) o aumento da capacidade das organizações atraírem e manterem desempenho superior; 5) o aumento da consistência do desempenho e 6) a adaptação dos funcionários às mudanças inerentes ao ambiente de trabalho (Podsakoff e Mackenzie, 1997).

A relevância do comportamento de cidadania organizacional está no fato de os funcionários realizarem atividades que vão além de suas funções, portanto exercem ações "extrapapel", contribuindo, assim, para o desempenho organizacional (Çinar, Karcioglu e Aliogullari, 2013). Conforme Podsakoff, Mackenzie, Paine e Bachrach (2000), esse comportamento contribui para obter mais: produtividade, liberação de recursos produtivos, desenvolvimento de atividades em grupo, redução de manutenção, retenção dos melhores funcionários, desempenho organizacional e adaptação eficaz às mudanças ambientais.

\subsection{Estudo de Burney, Henle e Widener (2009)}

A exposição do estudo de Burney, Henle e Widener (2009), publicado no periódico Accounting, Organizations and Society, deve-se ao fato de ele amparar esta pesquisa. Os autores analisaram a percepção de justiça organizacional (justiça processual e justiça distributiva) e comportamento de cidadania organizacional com o SPMS, utilizado para pagamento de incentivos. Os resultados indicaram que a percepção de justiça organizacional pode ser utilizada para a aceitabilidade dos sistemas de incentivos, contribuindo para a melhoria do desempenho dos empregados.

0 estudo foi realizado em uma organização do setor financeiro, um banco europeu, tendo como população da pesquisa os 540 funcionários distribuídos em 47 filiais, e a amostra final resultou em 242 respostas válidas. Instruções sobre a pesquisa foram transmitidas aos gerentes que repassaram as informações aos seus subordinados. 0 questionário foi estruturado de modo a identificar a percepção dos funcionários quanto ao grau de validade técnica, extensão reflexiva do modelo estratégico causal, justiça organizacional, comportamento de cidadania organizacional e desempenho.

Para a estimação do modelo teórico, utilizou-se a técnica de modelagem de equações estruturais e o software AMOS 4.0. Pelo modelo, constatou-se que percepções mais elevadas de justiça processual estão associadas com maior desempenho do funcionário por meio 
de comportamento de cidadania organizacional. Além disso, observou-se que o banco não precisa inserir aspectos subjetivos no SPMS para assegurar percepção positiva dos funcionários, e a postura da instituição, a fim de otimizar o desempenho, deve ser direcionada para aspectos relativos à percepção de justiça organizacional dos empregados.

Como limitações, os autores ressaltaram que os dados foram coletados a partir da percepção de funcionários e supervisores, o que torna a pesquisa subjetiva. Recomendam-se futuras pesquisas triangular os dados com outras fontes de pesquisa ou realizar estudos experimentais. Portanto, a presente investigação diferencia-se do estudo original no que concerne à organização foco de estudo, que no caso é uma cooperativa de crédito, por ser orientada por princípios cooperativos.

\section{Metodologia da pesquisa}

Este estudo descritivo, com abordagem quantitativa, foi realizado por meio de uma survey online. A natureza descritiva do estudo decorre do fato de se descrever a relação da percepção de justiça organizacional com o comportamento de cidadania organizacional de empregados na perspectiva do SPMS.

\subsection{Hipóteses de pesquisa}

Burney, Henle e Widener (2009) destacam que o SPMS precisa estar acessível, transparente e ser de fácil compreensão aos funcionários. Esses autores advertem ainda que, à medida que o SPMS reflete um modelo estratégico causal, ocorre a comunicação entre as ações dos funcionários e os objetivos organizacionais. Nesse sentido, Burney, Henle e Widener (2009) estabeleceram a seguinte hipótese: $\mathrm{H1a}$ : à medida que os empregados percebem que o SPMS reflete um modelo estratégico causal, ocorre a associação positiva com a justiça processual.

Se o SPMS contiver um elevado grau de validade técnica, as informações serão oportunas, compreensíveis e confiáveis (Burney, Henle e Widener, 2009). Um sistema complexo e inteligível facilita a comunicação das normas de avaliação dos empregados, dos objetivos organizacionais e do fornecimento de feedback. Portanto, se o SPMS estiver alinhado com a estratégia e apresentar elevado grau de validade técnica, contribuirá para capacitar os empregados e favorecerá sua percepção sobre o controle e a valorização atribuída pela organização (Burney, Henle e Widener, 2009). Assim, formularam a seguinte hipótese: 
H1b: ao passo que os empregados percebem que o SPMS é tecnicamente válido, ocorre a associação positiva com a justiça processual.

A validade técnica do SPMS fornece ao empregado informações precisas, oportunas, relevantes, compreensíveis e acessíveis. Essas informações serão a base da remuneração de incentivos dos empregados. Portanto, se o SPMS retratar de modo adequado a estratégia do modelo causal e apresentar grau de validade técnica, a percepção dos empregados sobre a distribuição das recompensas será associada com níveis mais elevados de justiça distributiva. Dessa forma, estabeleceram as seguintes hipóteses:

H2a: à proporção que os empregados percebem que o SPMS reflete um modelo estratégico causal, dá-se a associação positiva com a justiça distributiva;

H2b: à medida que os empregados percebem que o SPMS é tecnicamente válido, sucede a associação positiva com a justiça distributiva.

As percepções de justiça processual e de justiça distributiva geralmente estão relacionadas (Cropanzano e Ambrose, 2001). Segundo Colquitt et al. (2001), se os funcionários acreditam que sua remuneração é justa, possivelmente eles também julgam que os procedimentos são justos. Com base nessas assertivas Burney, Henle e Widener (2009) formularam a seguinte hipótese:

H3: a justiça distributiva está positivamente associada com a justiça processual.

Johnson, Selenta e Lord (2006) argumentam que a justiça organizacional se relaciona com comportamento de cidadania organizacional, já que o conceito de justiça organizacional fundamenta-se na percepção dos empregados sobre satisfação laboral, comprometimento organizacional, comportamento de cidadania organizacional e desempenho no trabalho.

Estudos empíricos fundamentam a relação entre comportamento de cidadania organizacional e desempenho no trabalho (Podsakoff, Ahearbe e Mackenzie, 1997). Segundo Burney, Henle e Widener (2009), a literatura suporta a hipótese de que a justiça processual afeta o comportamento de cidadania organizacional e, por sua vez, impacta no desempenho. Tais argumentos possibilitam os autores delinearem esta hipótese:

H4: a justiça processual está positivamente associada com o desempenho do empregado por meio do comportamento de cidadania organizacional. 
Dessa forma, a justiça processual será associada com o comportamento de cidadania organizacional, que se refere às atitudes dos empreegados para além das exigências do trabalho (Burney, Henle e Widener, 2009).

\section{Modelo teórico}

Na Figura 1, apresenta-se o modelo teórico que consubstanciou o estudo de Burney, Henle e Widener (2009) e que foi replicado nesta pesquisa.

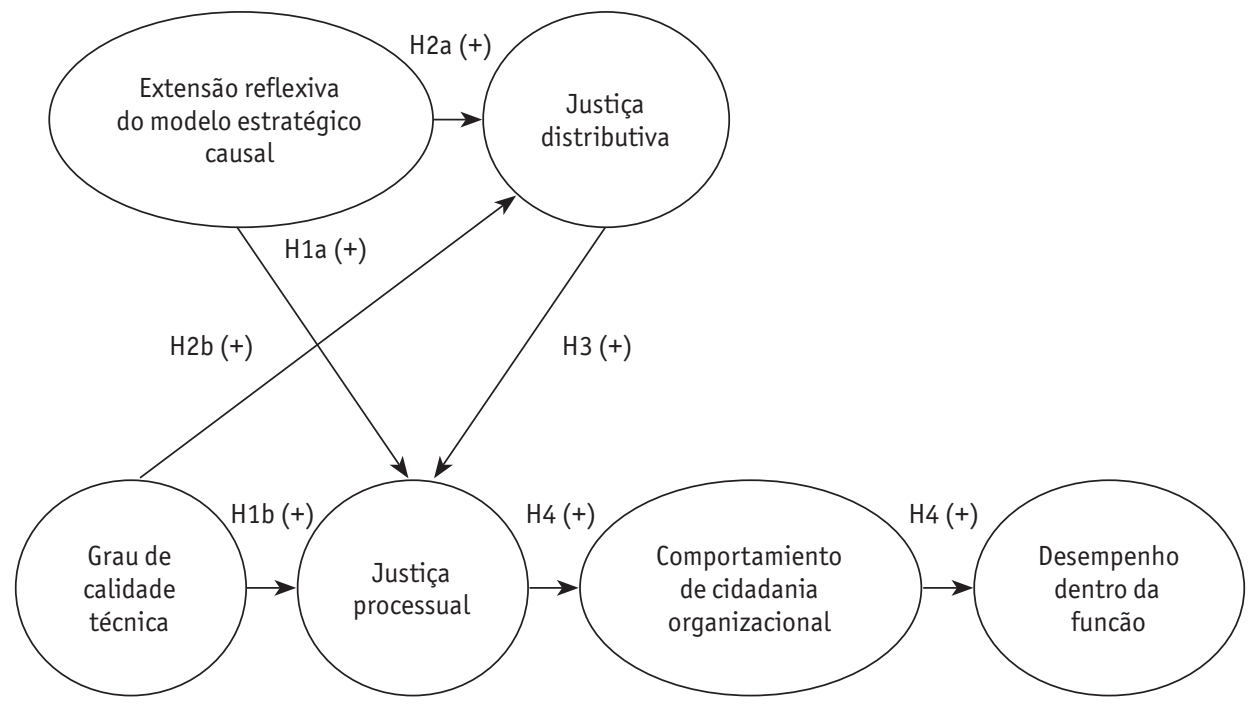

Figura 1. Modelo teórico

Fonte: Burney, Henle e Widener (2009, p. 307).

Observa-se, na Figura 1, o modelo teórico com a representação das relações de causa e efeito entre as variáveis que estão sendo investigadas. Salienta-se que as hipóteses desta pesquisa foram testadas com base nesse modelo teórico. 0 instrumento de pesquisa do estudo de Burney, Henle e Widener (2009) foi estruturado de modo que as variáveis assumissem modelos de medição reflexiva e fosse possivel testar as hipóteses teóricas.

\subsection{Construto da pesquisa}

Na Tabela 1, destacam-se as dimensões, as variáveis, o número de questões e as referências de suporte teórico e operacional do construto da pesquisa. 


\section{Tabela 1}

Construto da pesquisa

\begin{tabular}{|c|c|c|c|}
\hline Variáveis & Dimensões & $\begin{array}{c}\text { N. de itens } \\
\text { questionados }\end{array}$ & Referências \\
\hline Grau de validade técnica & $\begin{array}{l}\text { As informações fornecidas pelo SMDE são precisas, } \\
\text { acessiveis, confiáveis, oportunas e compreensíveis }\end{array}$ & 5 & McGowan's (1998) \\
\hline \multirow{3}{*}{$\begin{array}{l}\text { Extensão reflexiva do } \\
\text { modelo estratégico } \\
\text { causal }\end{array}$} & 0 SMDE incentiva a inclusão de medidas & 3 & \multirow{3}{*}{$\begin{array}{l}\text { Kaplan e Norton } \\
\text { (1996) }\end{array}$} \\
\hline & 0 SMDE me ajuda a entender & 4 & \\
\hline & $\begin{array}{l}\text { O SMDE contribui na tomada de decisões e na } \\
\text { avaliação de desempenho }\end{array}$ & 7 & \\
\hline \multirow{2}{*}{ Justiça organizacional } & Justiça distributiva & 4 & Colquitt (2001) \\
\hline & Justiça processual & 7 & Leventhal (1980) \\
\hline $\begin{array}{l}\text { Comportamento de cida- } \\
\text { dania organizacional }\end{array}$ & Comportamento de cidadania organizacional & 7 & $\begin{array}{l}\text { Williams e Anderson } \\
\text { (1991) }\end{array}$ \\
\hline $\begin{array}{l}\text { Desempenho dentro da } \\
\text { função }\end{array}$ & Desempenho dentro da função & 7 & $\begin{array}{l}\text { Williams e Anderson } \\
\text { (1991) }\end{array}$ \\
\hline
\end{tabular}

Fonte: elaboração própria com base em Burney, Henle e Widener (2009).

0 instrumento de pesquisa constituiu-se de um questionário estruturado com 44 questões, que contemplam as variáveis e dimensões do construto (Leventhal, 1980; Kaplan e Norton, 1996; Mcgowan's, 1998; Colquitt, 2001). As questões foram apresentadas em escala Likert de 7 pontos, conforme o estudo original. 0 questionário foi traduzido e submetido a um pré-teste para eliminar possíveis inconsistências e minimizar as dificuldades de compreensão. Para a realização do pré-teste, o instrumento de pesquisa foi aplicado a três profissionais com perfil semelhante ao da amostra. Além disso, os construtos foram validados com o coeficiente alpha de Cronbach.

\subsection{População e amostra}

A população da pesquisa compreende uma cooperativa de crédito, que possui remuneração baseada no SPMS. Justifica-se a realização do estudo em uma cooperativa de crédito devido a que as cooperativas de crédito são equiparadas no Brasil às instituições financeiras (Lei 4.595/1964) e seu funcionamento é legalizado pelo Banco Central. Além disso, prestam serviços de natureza bancária e de assistência creditícia, tendo como diferencial que os próprios cooperados exercem o papel de donos e usuários.

Com relação à remuneração fundamentada no SPMS, esse sistema é composto por medidas financeiras e não financeiras, portanto possibilita a emissão de relatórios (Gimbert, Bisbe e Mendoza, 2010; Bisbe e Malagueno, 2012) que poderão contribuir para a identificação 
de fatores críticos relacionados ao desempenho. A identificação dos pontos fracos pode motivar os empregados a terem melhor desempenho futuro.

Com base em uma lista de empregados de algumas unidades da cooperativa de crédito em estudo, enviou-se o questionário. Como amostra final, obtiveram-se 105 respostas válidas; portanto, trata-se de uma amostra não intencional, por acessibilidade.

\subsection{Coleta e análise dos dados}

Para a coleta de dados, o contato com a cooperativa se deu por meio de e-mails ao departamento de Recursos Humanos, nos quais se esclareceu o propósito do estudo. 0 instrumento de pesquisa foi encaminhado aos empregados pelo Google Docs em julho de 2014 . Ressalta-se que o consentimento à pesquisa foi realizado à medida que os empregados responderam ao questionário. Ainda, foi assegurado aos respondentes o anonimato.

Na análise dos dados, utilizou-se a modelagem de equações estruturais (Structural Equation Modeling: SEM). Klem (1995) considera essa técnica como uma extensão da regressão múltipla, pois na regressão se prevê uma única variável dependente, enquanto na modelagem de equações estruturais há mais de uma variável dependente. De acordo com Hair Jr, Black, Babin, Anderson e Tatham (2009), essa técnica estatística multivariada pode ser utilizada para elaborar modelos e ainda atuar de forma complementar aos métodos estatísticos tradicionais. Assim, a SEM possibilita ao pesquisador testar o efeito de causalidade entre as variáveis inseridas no modelo do estudo.

A SEM classifica-se em dois tipos: 1) modelagem de equações estruturais baseada em covariância ou modelo Lisrel e 2) mínimos quadrados parciais ou Partial Least Squares: PLS (Bido, Silva, Souza e Godoy, 2010). A abordagem Lisrel busca testar modelos teóricos, enquanto o PLS centra-se na construção de modelos teóricos em uma perspectiva exploratória (Bido et al. , 2010). Nesta pesquisa, utilizou-se a técnica PLS em decorrência da possibilidade de utilizar variáveis latentes com indicadores formativos em detrimento aos indicadores reflexivos (Hair, Ringle e Sarstedt, 2011). Os dados obtidos foram organizados no Microsoft Excel e utilizou-se o SmartPLS 2.0 para o seu processamento

\subsection{Descrição e análise dos resultados}

A Tabela 2 retrata o perfil dos respondentes quanto a gênero, faixa etária, tempo que trabalha na cooperativa, escolaridade, área de formação e nível hierárquico. 


\section{Tabela 2}

Perfil dos respondentes da pesquisa

\begin{tabular}{|c|c|c|c|}
\hline Gênero & $\%$ & Escolaridade & $\%$ \\
\hline Masculino & 55 & Graduação* & 74 \\
\hline Feminino & 45 & Pós-graduação*** & 45 \\
\hline Faixa etária & $\%$ & Área de formação & $\%$ \\
\hline Até 20 anos & 5 & Ciências contábeis & 35 \\
\hline De 21 a 30 anos & 63 & Administração & 29 \\
\hline De 31 a 40 anos & 27 & Economia & 14 \\
\hline De 41 a 50 anos & 3 & Outros & 22 \\
\hline Acima de 50 anos & 2 & & \\
\hline Tempo que trabalha na cooperativa & $\%$ & Nível hierárquico & $\%$ \\
\hline Até 5 anos & 67 & Colaborador & 72 \\
\hline De 6 a 10 anos & 22 & Gerente & 24 \\
\hline De 11 a 15 anos & 8 & Supervisor/coordenador & 2 \\
\hline \multirow[t]{2}{*}{ De 16 a 20 anos } & 3 & Diretor & 1 \\
\hline & & Presidente & 1 \\
\hline
\end{tabular}

* Da amostra, $74 \%$ possuem graduação e $26 \%$ não possuem graduação.

** Dos que possuem graduação, $45 \%$ concluíram algum curso de pós-graduação.

Fonte: elaboração própria.

Observa-se, na Tabela 2, que $55 \%$ são do gênero masculino e $45 \%$ feminino, o que indica equidade na representatividade entre os respondentes. A maioria (63\%) enquadra-se na faixa etária de 21 a 30 anos, seguida da faixa de 31 a 40 anos (27 \%). 0 tempo de trabalho na cooperativa ficou com o maior escore para até 5 anos. Observa-se um baixo nível de rotatividade do pessoal, tendo em vista que $22 \%$ dos empregados atuam na cooperativa há mais de 6 anos. Com relação à escolaridade, $74 \%$ possuem graduação e $45 \%$ pós-graduação em nível de especialização. Dos graduados, 35 \% são formados em ciências contábeis, $29 \%$ em administração e $14 \%$ em economia. Quanto ao nível hierárquico, a participação no estudo deu-se em diferentes níveis, mas com maior representatividade (72\%) dos colaboradores, seguido pelos gerentes (24\%).

\subsection{Modelagem de equações estruturais}

Além da estatística descritiva, utilizou-se a modelagem de equações estruturais por meio da técnica Partial Least Squares: PLS. A amostra compreendeu 105 observações, 6 variáveis latentes, 7 interações e 44 indicadores. Para realizar os testes estatísticos do modelo estrutural, analisaram-se a validade convergente, a confiabilidade e a validade discriminante. 
Tabela 3

Teste estatístico do modelo

\begin{tabular}{|c|c|c|c|c|}
\hline Variáveis latentes & $\begin{array}{l}\text { Coeficiente } \\
\text { R-Squared }\end{array}$ & $\begin{array}{c}\text { Coeficiente de } \\
\text { confiabilidade composta }\end{array}$ & $\begin{array}{l}\text { Coeficiente alpha } \\
\text { de Cronbach }\end{array}$ & $\begin{array}{l}\text { Variância média } \\
\text { explicada }\end{array}$ \\
\hline $\begin{array}{l}\text { Comportamento de cidadania } \\
\text { organizacional }\end{array}$ & 0,0783 & 0,6943 & 0,5310 & 0,2866 \\
\hline Desempenho dentro da função & 0,3553 & 0,7774 & 0,6693 & 0,3621 \\
\hline $\begin{array}{l}\text { Extensão reflexiva do modelo } \\
\text { estratégico causal }\end{array}$ & - & 0,9517 & 0,9449 & 0,5862 \\
\hline Grau de validade técnica & - & 0,9318 & 0,9087 & 0,7321 \\
\hline Justiça distributiva & 0,2949 & 0,9541 & 0,9357 & 0,8386 \\
\hline Justiça processual & 0,6751 & 0,9253 & 0,9058 & 0,6389 \\
\hline
\end{tabular}

Fonte: elaboração própria.

Inicialmente, observa-se a validade convergente (VC) do modelo, obtida pela variância média explicada VME. 0 critério de análise baseia-se em Fornell e Larcker (1981), que estabeleceram que os valores obtidos pela VME devem ser superiores a 0,50 (VME >0,50). Na Tabela 3, constata-se que as variáveis latentes, a extensão reflexiva do modelo estratégico causal, o grau de validade técnica, a justiça distributiva e a justiça processual apresentaram validade discriminante acima de 0,50; dessa forma, o modelo converge a um resultado satisfatório. Ressalta-se que dois construtos (comportamento de cidadania organizacional $=0,2866$ e desempenho dentro da função $=0,3621$ ) apresentaram VME<0,50; não obstante, prosseguiu-se à análise considerando as pesquisas anteriores (Leventhal, 1980; Williams e Anderson, 1991; Kaplan e Norton, 1996; Mcgowan's, 1998; Colquitt, 2001) e ponderou-se que este estudo é uma replicação; desse modo, preservou-se a construção do modelo original.

Após constatar a VME, analisa-se a consistência interna por meio do alpha de Cronbach (AC) e da confiabilidade composta (CC). Como parâmetro de análise, Hair Jr et al. (2009) estabelecem que valores de AC que variem entre 0,60 e 0,70 são considerados adequados. Para este estudo, quatro variáveis latentes apresentaram valores do coeficiente AC superiores a 0,90, o que está bem acima do recomendado por Hair Jr et al. (2009), portanto indica consistência interna. Observa-se que apenas um dos construtos (comportamento de cidadania organizacional) não atendeu a esse critério. Apesar disso, o construto de comportamento de cidadania organizacional foi mantido na análise, tendo em vista que ele é uma dimensão teórica do modelo estrutural de Burney, Henle e Widener (2009). 
No estudo replicado, os autores identificaram por meio do AC a seguinte consistência interna nos construtos: grau de validade técnica $=0,872$, justiça distributiva $=0,662$ e justiça processual $=0,793$. 0 s valores nesta investigação foram superiores, portanto apresentaram maior confiabilidade interna. Por outro lado, para as variáveis latentes comportamento de cidadania organizacional $(A C=0,722)$ e desempenho dentro da função $(A C=0,899)$ os $A C$ obtidos nesta investigação foram inferiores. Por fim, a extensão reflexiva do modelo estratégico causal apresentou valores semelhantes.

Analisando-se a consistência interna, verifica-se que os coeficientes de CC apresentaram comportamento similar aos do AC; porém, os valores foram ainda mais expressivos, em alguns casos superiores a 0,95. Para Fornell e Larcker (1981) e Chin (1998), uma CC maior ou igual a 0,70 é considerada aceitável. Ressalta-se que somente a variável latente comportamento de cidadania organizacional $(C C=0,6943)$ apresentou indicador inferior a 0,70, mas quando se arredonda o número, o critério de CC é atendido.

0 próximo passo consiste em avaliar a validade discriminante (VD) do modelo proposto, com o intuito de observar como os construtos são independentes uns dos outros (Hair Jr et al., 2009). Pelo critério de Gaski e Nevin (1985), confrontam-se os coeficientes de CC de cada variável latente com as correlações de Pearson, de modo que os valores das CC devem ser superiores aos das correlações entre os construtos. Pela Tabela 4 observa-se que para todos os construtos analisados os coeficientes de CC foram superiores às correlações de Pearson, portanto o modelo estrutural apresenta VD.

\section{Tabela 4}

Correlação de Pearson entre as variáveis latentes ou construtos

\begin{tabular}{|c|c|c|c|c|c|c|}
\hline Variáveis latentes & $\begin{array}{c}\text { Comportamento } \\
\text { de cidadania } \\
\text { organizacional }\end{array}$ & $\begin{array}{c}\text { Desempenho } \\
\text { dentro da } \\
\text { função }\end{array}$ & $\begin{array}{l}\text { Extensão reflexiva } \\
\text { do modelo } \\
\text { estratégico causal }\end{array}$ & $\begin{array}{c}\text { Grau de } \\
\text { validade } \\
\text { técnica }\end{array}$ & $\begin{array}{c}\text { Justiça } \\
\text { distributiva }\end{array}$ & $\begin{array}{c}\text { Justiça } \\
\text { processual }\end{array}$ \\
\hline $\begin{array}{l}\text { Comportamento de cidadania } \\
\text { organizacional }\end{array}$ & 0,6943 & & & & & \\
\hline Desempenho dentro da função & 0,5961 & 0,7774 & & & & \\
\hline $\begin{array}{l}\text { Extensão reflexiva do modelo } \\
\text { estratégico causal }\end{array}$ & 0,2338 & 0,2532 & 0,9517 & & & \\
\hline Grau de validade técnica & 0,2088 & 0,2463 & 0,8515 & 0,9318 & & \\
\hline Justiça distributiva & 0,1862 & 0,1064 & 0,5430 & 0,4619 & 0,9541 & \\
\hline Justiça processual & 0,2799 & 0,3032 & 0,7619 & 0,7422 & 0,6241 & 0,9253 \\
\hline
\end{tabular}

Fonte: elaboração própria. 
Na Tabela 5, retratam-se as relações dos coeficientes estimados dos caminhos do modelo estrutural e os níveis de significância.

\section{Tabela 5}

Relação das hipóteses

\begin{tabular}{|c|c|c|c|c|c|}
\hline Hipóteses & $\begin{array}{c}\text { Coeficiente } \\
\text { Beta }\end{array}$ & Erro Padrão & Valor de $\mathrm{t}$ & P-value & $\begin{array}{c}\text { Relação } \\
\text { Direta }\end{array}$ \\
\hline $\begin{array}{l}\text { H1a: extensão reflexiva do modelo estratégico causal } \\
\text {-> justiça processual }\end{array}$ & 0,3100 & 0,1763 & 0,1763 & 0,0790 & Sig. ${ }^{*}$ \\
\hline H1b: grau de validade técnica -> justiça processual & 0,3403 & 0,1527 & 2,2280 & 0,0261 & Sig. ${ }^{* *}$ \\
\hline $\begin{array}{l}\text { H2a: extensão reflexiva do modelo estratégico causal } \\
\text {-> justiça distributiva }\end{array}$ & 0,5448 & 0,1838 & 2,9635 & 0,0031 & Sig. ${ }^{* * *}$ \\
\hline H2b: Grau de validade técnica -> justiça distributiva & $-0,0020$ & 0,2200 & 0,0091 & 0,9927 & Não sig. \\
\hline H3: justiça distributiva -> justiça processual & 0,2986 & 0,0861 & 3,4682 & 0,0005 & Sig. ${ }^{* * *}$ \\
\hline $\begin{array}{l}\text { H4: justiça processual -> comportamento de cidadania } \\
\text { organizacional }\end{array}$ & 0,2799 & 0,1087 & 2,5753 & 0,0102 & Sig. ${ }^{* * *}$ \\
\hline $\begin{array}{l}\text { H4: comportamento de cidadania organizacional -> } \\
\text { desempenho dentro da função }\end{array}$ & 0,5961 & 0,0729 & 8,1735 & 0,0000 & Sig. ${ }^{* * *}$ \\
\hline
\end{tabular}

${ }^{*} p<0,10{ }^{* *} p<0,05 * * * p<0,01$

Fonte: elaboração própria.

Na Tabela 6, apresenta-se o resultado da regressão PLS, em que se destacam os coeficientes dos caminhos e os valores de $\mathrm{R}^{2}$.

\section{Tabela 6}

Resultado da regressão PLS: coeficiente dos caminhos, $p$-value $e R^{2}$

\begin{tabular}{|c|c|c|c|c|}
\hline \multirow[b]{2}{*}{ De } & \multicolumn{4}{|c|}{ Para } \\
\hline & $\begin{array}{c}\text { Justiça } \\
\text { distributiva }\end{array}$ & $\begin{array}{c}\text { Justiça } \\
\text { processual }\end{array}$ & $\begin{array}{c}\text { Comportamento } \\
\text { de cidadania } \\
\text { organizacional }\end{array}$ & $\begin{array}{c}\text { Desempenho } \\
\text { dentro da função }\end{array}$ \\
\hline Extensão reflexiva do modelo estratégico causal & 0,5448 & 0,3100 & & \\
\hline Grau de validade técnica & $-0,0020$ & 0,3403 & & \\
\hline Justiça distributiva & & 0,2986 & & \\
\hline Justiça processual & & & 0,2799 & \\
\hline Comportamento de cidadania organizacional & & & & 0,5961 \\
\hline $\mathrm{R}^{2}$ & 0,2949 & 0,6751 & 0,0783 & 0,3553 \\
\hline
\end{tabular}

Fonte: elaboração própria.

Constata-se relação significativa entre a extensão reflexiva do modelo estratégico causal com a justiça processual $(0,3100, \mathrm{p}<0,10)(\mathrm{H} 1 \mathrm{a})$ e a justiça distributiva $(0,5448, \mathrm{p}<0,01)$ 
(H2a); do grau de validade técnica com a justiça processual $(0,3403, \mathrm{p}<0,05)(\mathrm{H} 1 \mathrm{~b})$; da justiça distributiva com a justiça processual $(0,2986, \mathrm{p}<0,01)(\mathrm{H} 3)$; da justiça processual com o comportamento de cidadania organizacional $(0,2799, \mathrm{p}<0,01)(\mathrm{H} 4)$ e do comportamento de cidadania organizacional com o desempenho dentro da função $(0,5961, p<0,01)$ (H4). Todas essas relações apresentaram coeficiente Beta positivo, conforme Tabela 5.

Apenas a hipótese $\mathrm{H} 2 \mathrm{~b}$ não apresentou significância entre o grau de validade técnica e a justiça distributiva (-0,0020, Não sig.); portanto, a hipótese H2b não foi aceita, evidenciando que a percepção da validade técnica do SPMS não está associada com a justiça distributiva para os empregados da cooperativa de crédito. Assim, estes não consideram a relação entre informações precisas, acessiveis, confiáveis, oportunas e compreensíveis do SPMS com aspectos vinculados a remuneração, reconhecimento atribuído à execução de atividades e/ou ao desempenho no trabalho.

Os resultados deste estudo convergem com os de Burney, Henle e Widener (2009), ressalvada a hipótese $\mathrm{H} 2 \mathrm{~b}$, que no estudo desses autores foi aceita, de modo que todas as outras hipóteses estabelecidas no estudo base foram confirmadas. Esses autores constataram que a extensão reflexiva do modelo estratégico causal influencia positivamente a justiça processual $(0,179, \mathrm{p}<0,05)$ ( $\mathrm{H} 1 \mathrm{a})$ e a justiça distributiva $(0,312, \mathrm{p}<0,01)(\mathrm{H} 2 \mathrm{a}) ;$ o grau de validade técnica está associado positivamente com a justiça processual $(0,365, p<0,01)$ (H1b) e a justiça distributiva $(0,257, \mathrm{p}<0,05)(\mathrm{H} 2 \mathrm{~b})$; a justiça distributiva reflete-se positivamente na justiça processual $(0,422, \mathrm{p}<0,001)(\mathrm{H} 3)$; a justiça processual influencia positivamente no comportamento de cidadania organizacional $(0,169, \mathrm{p}<0,05)(\mathrm{H} 4)$ e 0 comportamento de cidadania organizacional interage positivamente com o desempenho dentro da função $(0,793, p<0,01)(H 4)$. Na Figura 2, evidencia-se o modelo empírico obtido pelo PLS.

Para os construtos endógenos, verifica-se por meio do $\mathrm{R}^{2}$, Tabela 6 e Figura 2, o ajuste do modelo para cada equação estrutural. $0 \mathrm{R}^{2}$ indica a porcentagem da variância de um construto que é explicado por outros construtos (Hair Jr et al., 2009). 0 menor $\mathrm{R}^{2}$ entre as variáveis latentes foi 0,0783 para comportamento de cidadania organizacional, apresentando um efeito pequeno (Cohen, 1977), mas consistente ao modelo estrutural de Burney, Henle e Widener (2009), que encontrou para essa relação um $R^{2}$ de 0,028 . Para a justiça processual e a justiça distributiva, os $\mathrm{R}^{2}$ foram mais expressivos, apresentando efeitos grandes (Cohen, 1977) e similares aos do estudo de Burney, Henle e Widener (2009), que encontraram um $R^{2}=0,652$ para justiça processual e um $R^{2}=0,274$ para justiça distributiva. 


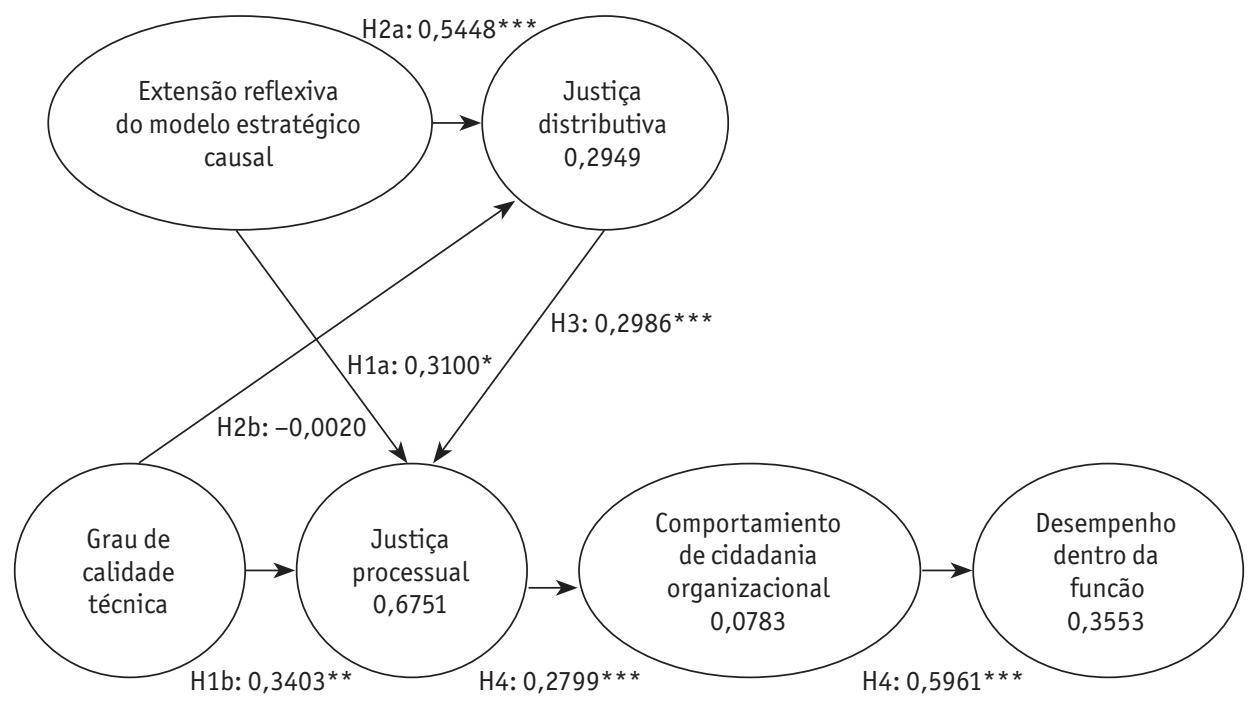

Figura 2. Modelo empírico obtido pelo PLS

${ }^{*} p<0,10 * * p<0,05 * * * p<0,01$

Fonte: elaboração própria.

\section{Conclusões}

0 estudo objetivou verificar a relação entre justiça organizacional e comportamento de cidadania organizacional de empregados na perspectiva do SPMS de uma cooperativa de crédito. Na estatística descritiva, utilizada para a análise dos dados demográficos, destaca-se que os respondentes da pesquisa apresentam-se equitativos quanto ao gênero, são em sua maioria jovens, com baixo nível de rotatividade no emprego, a maioria possui graduação e quase a metade curso de pós-graduação, e são de diferentes níveis hierárquicos, com predominância de colaboradores (empregados com funções operacionais). Esse perfil está alinhado para o atendimento do propósito do estudo.

Para analisar as hipóteses, foi aplicada a modelagem de equações estruturais, na abordagem dos PLS. A partir do test-t, p-value e coeficiente Beta não se rejeitaram as seguintes hipóteses: extensão reflexiva do modelo estratégico causal para justiça processual (H1a); grau de validade técnica para justiça processual (H1b); extensão reflexiva do modelo estratégico causal para justiça distributiva (H2a); justiça distributiva para justiça processual (H3); justiça processual para comportamento de cidadania organizacional e 
comportamento de cidadania organizacional para desempenho dentro da função $(\mathrm{H} 4)$. Todas essas relações indicaram significância estatística nos resultados, o que converge com os resultados do estudo de Burney, Henle e Widener (2009).

Por outro lado, somente a relação entre o grau de validade do SPMS e a justiça distributiva não foi significante; logo, rejeita-se a hipótese $\mathrm{H} 2 \mathrm{~b}$, o que diverge do estudo base. Essa relação evidencia que, para os empregados da cooperativa de crédito, a legitimidade técnica do SPMS não reflete em melhores remunerações, promoções ou em otimização do desempenho no trabalho. Assim, conclui-se que os resultados desta pesquisa assemelham-se aos da realizada por Burney, Henle e Widener (2009) em um banco europeu, o que contribui para a consolidação teórica dos aspectos abordados, além de instigar mais pesquisas para explicar as diferenças encontradas.

Os resultados deste estudo contribuem para: 1) evidenciar a relevância do SPMS, com enfoque nos incentivos, no entendimento e na importância dele para a cooperativa de crédito; 2) ressaltar a percepção dos empregados com relação à justiça organizacional, nas dimensões da justiça processual, que se relaciona aos procedimentos internos adotados pela cooperativa, e da justiça distributiva, que reflete aspectos de compensações e remunerações atrelados ao desempenho no trabalho; 3) salientar o comportamento de cidadania organizacional, que se refere à atuação dos empregados no que tange ao comprometimento com o trabalho e 4) identificar a postura dos empregados para realizar as suas tarefas, de modo a observar o seu desempenho no trabalho.

Todavia, na análise dos resultados, deve-se ponderar que as respostas fundamentam-se na percepção dos respondentes. Desse modo, os resultados poderiam ser diferentes se a amostra investigada integrasse outro ramo de atuação, outro porte de empresa ou ainda se os aspectos culturais exercessem forte influência. Ainda, como limitação, salienta-se que o modelo estrutural utilizado foi construído a partir de conceitos teóricos; logo, é possível que indicadores relevantes não tenham integrado os construtos definidos.

Recomenda-se que pesquisas futuras sejam realizadas em outras organizações ou setores econômicos a fim de validar a construção teórica utilizada e expandir as pesquisas que utilizam a técnica de equações estruturais. Além disso, pode-se replicar este estudo e inserir variáveis de controle, como porte da cooperativa, por exemplo. Outra sugestão é a constatação in loco sobre a falta de significância para a relação da validade técnica do SPMS quando associado com a justiça distributiva. 


\section{Referências}

Adams, J. S. (1965). Inequity in social exchange. Advances in experimental social psychology, $2(1), 267-299$.

Aracioglu, B., Zalluhoglu, A. E., and Candemir, C. (2013). Measuring and evaluating performance within the strategic management perspective: A study on performance measurement of a seafood company. Procedia-Social and Behavioral Sciences, 99 (1), 1026-1034.

Assmar, E. M. L., Ferreira, M. C. e Souto, S. 0. (2005). Justiça organizacional: uma revisão crítica da literatura. Psicologia: reflexão e crítica, 18 (3), 443-453.

Beer, M., and Cannon, M. D. (2004). Promise and peril in implementing pay-for-performance. Human Resource Management, 43 (1), 3-48.

Bialoskorski Neto, S. e Marques, P. V. (1998). Agroindústria cooperativa: um ensaio sobre crescimento e estrutura de capital. Gestão e Produção, 5 (1), 60-68.

Bido, D. S., Silva, D., Souza, C. A. e Godoy, A. S. (2010). Mensuração com indicadores formativos nas pesquisas em administração de empresas: como lidar com a multicolinearidade entre eles? Administração: Ensino e Pesquisa, 11 (2), 245-269.

Bisbe, J., and Malagueno, R. (2012). Using strategic performance measurement systems for strategy formulation: Does it work in dynamic environments? Management Accounting Research, 23 (4), 296-311.

Burney, L. L., Henle, C. A., and Widener, S. K. (2009). A path model examining the relations among strategic performance measurement system characteristics, organizational justice, and extra-and in-role performance. Accounting, Organizations and Society, 34 (3), 305-321.

Chaneta, I. (2007). Strategic management process. Journal of Comprehensive Research, 5 (1), 17-25.

Chenhall, R. H. (2005). Integrative strategic performance measurement systems, strategic alignment of manufacturing, learning and strategic outcomes: An exploratory study. Accounting, Organizations and Society, 30 (5), 395-422.

Chin, W. W. (1998). The partial least squares approach to structural equation modeling. In Modern methods for business research. London: Lawrence Erlbaum.

Çinar, 0., Karcioglu, F., and Aliogullari, Z. D. (2013). The relationship between organizational silence and organizational citizenship behavior: A survey study in the Province of Erzurum, Turkey. Procedia-Social and Behavioral Sciences, 99 (1), 314-321.

Cohen, J. (1977). Statistical Power Analysis for the Behavioral Sciences. New York: Academic Press. Colquitt, J. A. (2001). On the dimensionality of organizational justice: A construct validation of a measure. Journal of Applied Psychology, 86 (3), 386-400. 
Colquitt, J. A., Conlon, D. E., Wesson, M. J., Porter, C. O. L. H., and Ng, K. Y. (2001). Justice at the millennium: A meta-analytic review of 25 years of organizational justice research. Journal of Applied Psychology, 86 (3), 425-445.

Crabtree, A. D., and Debusk, G. K. (2008). The effects of adopting the balanced scorecard on shareholder returns. Advances in Accounting , 24 (1), 8-15.

Cropanzano, R., and Ambrose, M. L. (2001). Procedural and distributive justice, are more similar than you think: A monistic perspective and a research agenda. In Advances in organizational justice (pp. 119-151). Stanford, CA: Stanford University Press.

Erdogan, B. (2002). Antecedents and consequences of justice perceptions in performance appraisals. Human Resource Management Review, 12 (4), 555-578.

Folger, R., and Cropanzano, R. (1998). Organizational Justice and Human Resource Management. Thousand Oaks, VA: Sage.

Folger, R., Konovsky, M. A., and Cropanzano, R. (1992). A due process metaphor for performance appraisal. Research in Organizational Behavior, 14 (1), 129-177.

Fornell, C., and Larcker, D. F. (1981). Evaluating structural equation models with unobservable variables and measurement error. Journal of Marketing Research, 18 (1), 39-50.

Franco-Santos, M., Lucianetti, L., and Bourne, M. (2012). Contemporary performance measurement systems: A review of their consequences and a framework for research. Management Accounting Research, 23 (2), 79-119.

Garengo, P., Biazzo, S., and Bititci, U. S. (2005). Performance measurement systems in SMEs: A review for a research agenda. International Journal of Management Review, 7 (1), 25-47.

Gaski, J. F., and Nevin, J. R. (1985). The differential effects of exercised and unexercised power sources in a marketing channel. Journal of Marketing Research, 22 (2), 130-142.

Geuser, F., Mooraj, S., and Oyon, D. (2009). Does the Balanced Scorecard Add Value? Empirical evidence on its effect on performance. European Accounting Review, 18 (1), 93-122.

Gibbs, M., Merchant, K. A., Van Der Stede, W. A., and Vargus, M. E. (2004). Determinants and effects of subjectivity in incentives. The Accounting Review, 79 (2), 409-436.

Gilliland, S. W., and Langdon, J. C. (1998). Creating performance management Systems that promote perceptions of fairness. In Performance Appraisal: State of the Art in Practice (pp. 209-243). San Francisco: Jossey Bass.

Gimbert, X., Bisbe, J., and Mendoza, X. (2010). The role of performance measurement systems in strategy formulation processes. Long Range Planning, 43 (4), 477-497.

Hair Jr, J. F., Black, W. C., Babin, B. J., Anderson, R. E. e Tatham, R. L. (2009). Análise multivariada de dados. 6. ed. Porto Alegre: Bookman.

Hair Jr, J. F., Ringle, C. M., and Sarstedt, M. (2011). PLS-SEM: Indeed a silver bullet. The Journal of Marketing Theory and Practice, 19 (2), 139-152. 
Hall, M. (2008). The effect of comprehensive performance measurement systems on role clarity, psychological empowerment and managerial performance. Accounting, Organizations and Society, 33 (2), 141-163.

Hartman, S. J., Yrle, A. C., and Galle Jr, W. P. (1999). Procedural and distributive justice: Examining equity in a university setting. Journal of Business Ethics, 20 (4), 337-352.

Homans, G. (1961). Social behavior. New York: Harcourt, Brace e World.

Ittner, C. D., Larcker, D. F., and Meyer, M. W. (2003). Subjectivity and the weighting of performance measures: Evidence from a Balanced Scorecard. The Accounting Review, 78 (3), 725-758.

Ittner, C. D., Larcker, D. F., and Randall, T. (2003). Performance implications of strategic performance measurement in financial services firms. Accounting, Organizations and Society, 28 (7), 715-741.

Johnson, R. E., Selenta, C., and Lord, R. G. (2006). When organizational justice and the self-concept meet: Consequences for the organization and its members. Organizational Behavior and Human Decision Processes, 99 (2), 175-201.

Kaplan, R. S., and Norton, D. P. (1996). The Balanced Scorecard: Translating Strategy into Action. Boston, MA: Harvard Business School Press.

Klem, L. (1995). Path analysis. In Reading and understanding multivariate statistics (pp. 65-98). Washington, DC: American Psychological Association.

Kolehmainen, K. (2010). Dynamic strategic performance measurement systems: Balancing empowerment and alignment. Long Range Planning, 43 (4), 527-554.

Leventhal, G. S. (1980). What Should Be Done with Equity Theory? New Approaches to the Study of Fairness in Social Relationships. New York: Plenum Press.

Leventhal, G. S., Karuza, J., and Fry, W. R. (1980). Beyond fairness: A theory of allocation preferences. Justice and social interaction, 3 (1), 167-218.

Locke, E. A., and Latham, G. P. (1990). A theory of Goal Setting and Task Performance. Englewood Cliff, NJ: Prentice Hall.

Marinho, S. V., and Cagnin, C. (2014). The roles of FTA in improving performance measurement systems to enable alignment between business strategy and operations: Insights from three practical cases. Futures, 54 (1), 50-61.

McGowan, A. S. (1998). Perceived benefits of ABCM Implementation. Accounting Horizons, 12 (1), 31-50.

Organ, D. W. (1998). Organizational Citizenship Behavior: The Good Soldier Syndrome. Lexington, MA: Lexington Books.

Organização das Cooperativas Brasileiras (OCB). Cooperativismo. Recuperado em 11 jun. 2014 de http://www.ocb.org.br/site/ocb/

Otley, D. (1999). Performance management: A framework for management control systems research. Management Accounting Research, 10 (4), 363-382. 
Podsakoff, P. M., Ahearne, M., and Mackenzie, S. B. (1997). Organizational citizenship behavior and the quantity and quality of work group performance. Journal of Applied Psychology, $82(2), 262-270$.

Podsakoff, P. M., and Mackenzie, S. B. (1997). Impact of organizational citizenship behavior on organizational performance: A review and suggestion for future research. Human Performance, 10 (2), 133-151.

Podsakoff, P. M., Mackenzie, S. B., Paine, J. B. e Bachrach, D.G. (2000). Organizational citizenship behaviors: a critical review of the theoretical and empirical literature and suggestions for future research. Journal of Management, 26 (3), 513-563.

Popescu, A. M., and Deaconu, A. (2013). High-school, Organizational Citizenship Behavior Moderator. Procedia-Social and Behavioral Sciences, 92 (1), 735-740.

Rego, A. (2002). Comprometimento afetivo dos membros organizacionais: o papel das percepções de justiça. Revista de Administração Contemporânea, 6 (2), 209-241.

Robbins, S. A. (2003). Organizational Behavior. Upper Saddle River, NJ: Prentice Hall.

Schiemann, W. A., and Lingle, J. H. (1997). Seven Greatest Myths of Measurement. Management Review, 86 (1), 29-32.

Sotomayor, A. M. S. B. (2007). Avaliação de desempenho e compromisso organizacional: a perspectiva da justiça organizacional. Revista Universo Contábil, 3 (3), 87-100.

Van Dyne, L., Graham, J. W., and Dienesch, R. M. (1994). Organizational citizenship behavior: Construct redefinition, measurement, and validation. Academy of Management Journal, 37 (4), 765-802.

Williams, L. J., and Anderson, S. E. (1991). Job satisfaction and organizational commitment as predictors of organizational citizenship and in-role behaviors. Journal of Management, $17(3), 601-617$. 
